\title{
AMENABILITY PROPERTIES OF FOURIER ALGEBRAS AND FOURIER-STIELTJES ALGEBRAS: A SURVEY
}

\author{
NICO SPRONK \\ Department of Pure Mathematics, University of Waterloo \\ 200 University Ave. W., Waterloo, Ontario, N2L 3G1, Canada \\ E-mail: nspronk@uwaterloo.ca
}

\begin{abstract}
Let $G$ be a locally compact group, and let $\mathrm{A}(G)$ and $\mathrm{B}(G)$ denote its Fourier and Fourier-Stieltjes algebras. These algebras are dual objects of the group and measure algebras, $\mathrm{L}^{1}(G)$ and $\mathrm{M}(G)$, in a sense which generalizes the Pontryagin duality theorem on abelian groups. We wish to consider the amenability properties of $\mathrm{A}(G)$ and $\mathrm{B}(G)$ and compare them to such properties for $\mathrm{L}^{1}(G)$ and $\mathrm{M}(G)$. For us, "amenability properties" refers to amenability, weak amenability, and biflatness, as well as some properties which are more suited to special settings, such as the hyper-Tauberian property for semisimple commutative Banach algebras. We wish to emphasize that the theory of operator spaces and completely bounded maps plays an indispensable role when studying $\mathrm{A}(G)$ and $\mathrm{B}(G)$. We also show some applications of amenability theory to problems of complemented ideals and homomorphisms.
\end{abstract}

1. Introduction. This article, as the title literally suggests, is a survey of the amenability results around Fourier and Fourier-Stieltjes algebras which are known to the author. These are displayed in comparison to the results on group and measure algebras. In particular, I intend to highlight the indispensable role which operator spaces play in this theory.

The scope of this article has been purposely restricted to only amenability properties of Fourier and Fourier-Stieltjes algebras, and to the motivating results in their dual objects, group and measure algebras. There are, thus, clear omissions of discussions of Herz-FigàTalamanca algebras $\mathrm{A}_{p}(G)$, in general, and their amenability properties; and of locally compact quantum groups or even Kac algebras, though they do provide a convenient language for discussing the duality. However, limited liberty is taken to indicate some

2010 Mathematics Subject Classification: Primary 43-02, 43A30, 46H25, 46L07; Secondary 43A07, 43A20, 43A10, 43A77, 22D10, 46J20, 43A85.

Key words and phrases: amenability, Fourier algebra, Fourier-Stieltjes algebra, operator space, completely bounded map.

The paper is in final form and no version of it will be published elsewhere. 
relevant literature on such topics, as they relate to the present article. The scope of this article has been restricted mainly because the topic has reached a certain maturity, though there are still interesting open problems on Fourier and Fourier-Stieltjes algebras. Moreover, it is hoped that this limitation in scope improves coherence and depth, though at the cost of breadth.

We acknowledge that this is far from being the first survey of amenability problems in Fourier and Fourier-Stieltjes algebras. See surveys of Runde [67, 69] and Kaniuth and Lau [48]. However, the subject has advanced significantly, even since the latter of these surveys. Moreover, our focus differs from the foci of these articles, thus we offer a complement to them.

1.1. Some Banach algebras of harmonic analysis. Let $G$ be a locally compact group. The group and measure convolution algebras of $G$, are denoted by $\mathrm{L}^{1}(G)$ and $\mathrm{M}(G)$, respectively. These classical objects are defined in many texts; see the treatise 37] for definitions and historical details. These algebras represent complete invariants for the underlying group in the sense that if $H$ is another locally compact group for which $\mathrm{L}^{1}(G) \cong \mathrm{L}^{1}(H)$, or $\mathrm{M}(G) \cong \mathrm{M}(H)$, isometrically isomorphically, then $G \cong H$ isomorphically and homeomorphically, as shown by Wendel 82 . We may, and will, regard $\mathrm{L}^{1}(G)$ as the ideal in $\mathrm{M}(G)$ of measures which are absolutely continuous with respect to the Haar measure.

If $G$ is abelian, it admits a dual group $\widehat{G}$, and the Fourier-Stieltjes transform $\mu \mapsto \hat{\mu}$ : $\mathrm{M}(\widehat{G}) \rightarrow \mathcal{C}_{b}(G)$ (bounded continuous functions on $G$ ) restricts to the Gelfand transform $f \mapsto \hat{f}: \mathrm{L}^{1}(\widehat{G}) \rightarrow \mathcal{C}_{0}(G)$ (continuous functions vanishing at infinity). Their respective images $\mathrm{B}(G)$ and $\mathrm{A}(G)$, each endowed with the norm which makes the transform an isometry, are thus Banach algebras of functions, which are invariants for $G$. It follows from Jordan decomposition in $\mathrm{M}(\widehat{G})$, and Bochner's theorem, that $\mathrm{B}(G)$ is the span of continuous positive definite functions; and then a version of the Gelfand-Naimark-Segal construction shows that $\mathrm{B}(G)$ consists of the family of continuous matrix coefficients of all unitary representations on $G$. Since each element of $\mathrm{L}^{1}(G)$ factors as a pointwise product of two square-integrable functions, i.e. $\mathrm{L}^{1}(\widehat{G})=\mathrm{L}^{2}(\widehat{G}) \cdot \mathrm{L}^{2}(\widehat{G})$, it follows from the Plancherel theorem that $\mathrm{A}(G)=\mathrm{L}^{2}(G) * \mathrm{~L}^{2}(G)$.

Eymard [17] showed, using representation theory, how to construct generalizations of $\mathrm{A}(G)$ and $\mathrm{B}(G)$ for any locally compact group. The Fourier-Stieltjes algebra $\mathrm{B}(G)$ is the space of continuous matrix coefficients of unitary representations of $G: s \mapsto\langle\pi(s) \xi \mid \eta\rangle$ for any weak operator continuous unitary representation $\pi: G \rightarrow \mathcal{B}(\mathcal{H}), \xi, \eta \in \mathcal{H}$. The Fourier algebra $\mathrm{A}(G)$ is the space of matrix coefficients of the left regular representation: $s \mapsto\langle\lambda(s) f \mid g\rangle=\bar{g} * \check{f}(s), f, g \in \mathrm{L}^{2}(G)$, where $\lambda: G \rightarrow \mathcal{B}\left(\mathrm{L}^{2}(G)\right)$ is given by $\lambda(s) f(t)=$ $f\left(s^{-1} t\right)$, and $\check{f}(t)=f\left(t^{-1}\right)$. $\mathrm{B}(G)$ may be identified as the dual space of the universal $\mathrm{C}^{*}$-algebra $\mathrm{C}^{*}(G)$, of $G$, and this norm makes $\mathrm{B}(G)$ a Banach algebra, under pointwise operations. $\mathrm{A}(G)$ is a closed ideal of $\mathrm{B}(G)$ a fact which may be verified by showing that $\mathrm{A}(G)$ is generated by the compactly supported elements of $\mathrm{B}(G)$, or, alternatively, by an application of Fell's "absorption principle" [18]. These are semisimple commutative Banach algebras, and $G$ is the Gelfand spectrum of $\mathrm{A}(G)$. Walter [81] showed the analogue of Wendel's theorem: if $H$ is another locally compact group then $\mathrm{A}(G) \cong \mathrm{A}(H)$, or 
$\mathrm{B}(G) \cong \mathrm{B}(H)$, isometrically isomorphically, exactly when $G \cong H$ isomorphically and homeomorphically. Walter's result foreshadows the use of operator space methods, in that he employs Kadison's characterizations of surjective isometries between $\mathrm{C}^{*}$-algebras [46].

It will be useful to identify various subalgebras of $\mathrm{B}(G)$. We let $\mathrm{B}_{r}(G)$ denote the weak*-closure of $\mathrm{A}(G)$ in $\mathrm{B}(G)$. It is a result of Hulanicki [39] that $\mathrm{B}_{r}(G)=\mathrm{B}(G)$ if and only if $G$ is amenable. Let $\mathrm{A}_{f}(G)$ denote the norm-closed subspace of $\mathrm{B}(G)$ generated by matrix coefficients of continuous finite dimensional representations. Then $\mathrm{A}_{f}(G) \cong$ $\mathrm{A}\left(G^{a p}\right)$ where $G^{a p}$ is the almost periodic compactification of $G$. We note that if $G$ is abelian, $\mathrm{A}_{f}(G)$ is the image under the Fourier transform of the closed span of Dirac measures $\ell^{1}(\widehat{G})$ in $\mathrm{M}(\widehat{G})$. We let the Rajchman algebra be given by $\mathrm{B}_{0}(G)=\mathrm{B}(G) \cap \mathcal{C}_{0}(G)$. For any non-compact abelian group, it is known that $\mathrm{B}_{0}(G) \supsetneq \mathrm{A}(G)$; see $\S 6$ of $[32$.

1.2. Amenability and related properties. If $\mathcal{A}$ is a Banach algebra, a Banach space $\mathcal{X}$ is called a (contractive) Banach $\mathcal{A}$-bimodule if there are bounded (contractive) maps, a homomorphism $\mathcal{A} \rightarrow \mathcal{B}(\mathcal{X}): a \mapsto(x \mapsto a \cdot x)$ and an anti-homomorphism $\mathcal{A} \rightarrow \mathcal{B}(\mathcal{X}):$ $a \mapsto(x \mapsto x \cdot a)$, with commuting ranges. The adjoints of these maps make the dual space $\mathcal{X}^{*}$ into a dual Banach $\mathcal{A}$-bimodule. A linear map $D: \mathcal{A} \rightarrow \mathcal{X}$ is called a derivation if $D(a b)=a \cdot D(b)+D(a) \cdot b$ for $a, b$ in $\mathcal{A}$. Inner derivations are those of the form $D(a)=a \cdot x-x \cdot a$ for some $x$ in $\mathcal{X}$.

In his seminal memoir [42, Johnson defined $\mathcal{A}$ to be amenable if, for every dual Banach $\mathcal{A}$-bimodule $\mathcal{X}^{*}$, every bounded derivation $D: \mathcal{A} \rightarrow \mathcal{X}^{*}$ is inner. He further showed in [43] that amenability is equivalent to a certain averaging property: $\mathcal{A}$ is amenable if and only if it admits a bounded approximate diagonal, i.e. a bounded net $\left(d_{\alpha}\right)$ in $\mathcal{A} \otimes^{\gamma} \mathcal{A}$ (projective tensor product) such that

$$
m\left(d_{\alpha}\right) a, a m\left(d_{\alpha}\right) \rightarrow a \quad \text { and } \quad a \cdot d_{\alpha}-d_{\alpha} \cdot a \rightarrow 0
$$

where $m: \mathcal{A} \otimes^{\gamma} \mathcal{A} \rightarrow \mathcal{A}$ is the multiplication map $m(a \otimes b)=a b$, and $a \cdot(b \otimes c)=$ $(a b \times c),(b \otimes c) \cdot a=b \otimes(c a)$. Bounded approximate diagonals allow for a quantitative measurement of amenability: we say $\mathcal{A}$ is $C$-amenable if it admits a bounded approximate diagonal of norm at most $C$, and let the amenability constant of $\mathcal{A}, C_{\mathcal{A}}$ denote the infimum of such $C$. Bounded approximate diagonals are also useful in the following application of amenability due to Helemskiu (see the monograph [35] or [10]; the role of the bounded approximate diagonal is apparent in [66]): if $\mathcal{A}$ is amenable and $\mathcal{X}$ is a Banach $\mathcal{A}$-bimodule with a boundedly complemented subspace $\mathcal{Y}$, which is also an $\mathcal{A}$-bimodule, then there is a bounded projection $P: \mathcal{X}^{*} \rightarrow \mathcal{Y}^{\perp}$ which is an $\mathcal{A}$-bimodule map.

We say $\mathcal{A}$ is contractible or super-amenable if all bounded derivations into (not necessarily dual) $\mathcal{A}$-bimodules are inner. A contractible $\mathrm{C}^{*}$-algebra is necessarily finite dimensional (see [66], for example).

We say $\mathcal{A}$ is a dual Banach algebra if there is a closed $\mathcal{A}$-bimodule $\mathcal{A}_{*} \subset \mathcal{A}^{* *}$ such that $\left(\mathcal{A}_{*}\right)^{*} \cong \mathcal{A}$. If every weak*-weak* continuous derivation $D: \mathcal{A} \rightarrow \mathcal{X}^{*}$ is inner, we say $\mathcal{A}$ is Connes amenable. This terminology first appeared in [65], and honours Connes's version of amenability for von Neumann algebras.

A Banach algebra is weakly amenable if every bounded derivation $D: \mathcal{A} \rightarrow \mathcal{A}^{*}$ is inner. For the case of commutative $\mathcal{A}$, this terminology was coined in [4, where it was 
shown that any derivation $D: \mathcal{A} \rightarrow \mathcal{X}$ for symmetric $\mathcal{X}$ (right and left actions coincide) vanishes, exactly when $\mathcal{A}$ is weakly amenable. Given a character character $\chi: \mathcal{A} \rightarrow \mathbb{C}$, a non-zero linear functional $d \in \mathcal{A}^{*}$ such that $d(a b)=\chi(a) d(b)+d(a) \chi(b)$ is called a point derivation. If a non-zero derivation exists, the derivation $D: \mathcal{A} \rightarrow \mathcal{A}^{*}, D(a)=d(a) \chi$, shows that $\mathcal{A}$ cannot be weakly amenable.

We indicate some related properties due to Helemskin [35]. $\mathcal{A}$ is biprojective if there is a bounded $\mathcal{A}$-bimodule map $S: \mathcal{A} \rightarrow \mathcal{A} \otimes^{\gamma} \mathcal{A}$ such that $m \circ S=\mathrm{id}_{\mathcal{A}}$. $\mathcal{A}$ is biflat if there is a bounded $\mathcal{A}$-bimodule map $T:\left(\mathcal{A} \otimes^{\gamma} \mathcal{A}\right)^{*} \rightarrow \mathcal{A}^{*}$ such that $m^{*} \circ T=\operatorname{id}_{\mathcal{A}^{*}}$. A biflat algebra is automatically weakly amenable. We also have that $\mathcal{A}$ is amenable if and only if it is biflat and has a bounded approximate identity.

1.3. Commutative Banach algebras. We let, for this section, $\mathcal{A}$ be a regular function algebra on a locally compact space $X$, i.e. $\mathcal{A}$ is a semisimple commutative Banach algebra for which there is a contractive inclusion $\mathcal{A} \subset \mathcal{C}_{0}(X)$ whose image contains functions which separate compact sets from disjoint closed sets. If $E \subset X$ is closed we let

$$
\begin{gathered}
\mathrm{I}_{\mathcal{A}}(E)=\left\{u \in \mathcal{A}:\left.u\right|_{E}=0\right\}, \quad \mathrm{I}_{\mathcal{A}}^{c}(E)=\left\{u \in \mathrm{I}_{\mathcal{A}}: \operatorname{supp} u \text { is compact }\right\} \\
\text { and } \mathrm{I}_{\mathcal{A}}^{0}(E)=\left\{u \in \mathrm{I}_{\mathcal{A}}^{c}: \operatorname{supp} u \cap E=\varnothing\right\} .
\end{gathered}
$$

We note that $\mathrm{I}_{\mathcal{A}}^{0}(E)$ is the smallest ideal of elements of $\mathcal{A}$ which vanish on $E$ (see [62]), and $\mathrm{I}_{\mathcal{A}}(E)$ is the largest. We say that $E$ is a spectral set for $\mathcal{A}$ if $\overline{\mathrm{I}_{\mathcal{A}}^{0}(E)}=\mathrm{I}_{\mathcal{A}}(E)$, approximable if $\mathrm{I}_{\mathcal{A}}(E)$ has a bounded approximate identity, locally spectral if $\overline{\mathrm{I}_{\mathcal{A}}^{0}(E)} \supset \mathrm{I}_{\mathcal{A}}^{c}(E)$, and essential if $\overline{\mathrm{I}_{\mathcal{A}}(E)^{2}}=\mathrm{I}_{\mathcal{A}}(E)$. The Tauberian property for $\mathcal{A}$ is that $\varnothing$ is spectral for $\mathcal{A}$.

There is a notion of support of a functional $\mu$ in $\mathcal{A}^{*}$, which generalizes that for a measure. Using this, Samei [77] devised an amenability-type property for some Banach function algebras. $\mathcal{A}$ is called hyper-Tauberian if every bounded linear local map $T$ : $\mathcal{A} \rightarrow \mathcal{A}^{*}$, i.e $\operatorname{supp}(T u) \subset \operatorname{supp} u$ for $u$ in $\mathcal{A}$, is an $\mathcal{A}$-module map. He proved that the hyper-Tauberian condition implies weak amenability.

We have the following relationship between amenability conditions and spectral conditions.

Theorem 1.1. Let $\mathcal{A}$ be a regular function algebra on $X$ for which $\mathcal{A} \otimes^{\gamma} \mathcal{A}$ is semi-simple. Then $\mathcal{A} \otimes^{\gamma} \mathcal{A}$ is regular on $X \times X$ and $\mathrm{I}_{\mathcal{A} \otimes \gamma \mathcal{A}}(\Delta)=\operatorname{ker} m$ where $\Delta=\{(x, x): x \in X\}$. Moreover we have

(i) $\mathcal{A}$ is amenable if and only if $\varnothing$ is approximable for $\mathcal{A}$ and $\Delta$ is approximable for $\mathcal{A} \otimes{ }^{\gamma} \mathcal{A}$

(ii) $\mathcal{A}$ is hyper-Tauberian if and only if $\varnothing$ is spectral for $\mathcal{A}$ and $\Delta$ is locally spectral for $\mathcal{A} \otimes \otimes^{\gamma} \mathcal{A}$

(iii) if $\varnothing$ is approximable for $\mathcal{A}$, then $\mathcal{A}$ is weakly amenable if and only $\Delta$ is essential for $\mathcal{A} \otimes^{\gamma} \mathcal{A}$

(iii') $\mathcal{A}$ is weakly amenable if $\varnothing$ is essential for $\mathcal{A}$ and $\Delta$ is spectral for $\mathcal{A} \otimes^{\gamma} \mathcal{A}$.

We note that (i) is a specialized version of a famous splitting result of [35]; see also [10]. The result (ii) is a mild generalization of a result in [77]; there, the only extra assumption is that $X$ is the spectrum of $\mathcal{A}$. The result (iii) is in [34; (iii') is an easy corollary of the same theorem of [34] in which (iii) is stated. 
QUESTION 1.2. (i) Is it the case that amenability implies hyper-Tauberianness?

(ii) Is there an example of an approximable set which is not (locally) spectral?

A positive answer to (ii) would dismiss an obvious route to proving (i).

\section{Amenability properties of Banach algebras of harmonic analysis}

2.1. Group and measure algebras. Let $G$ be a locally compact group. The property of amenability for groups is well known; see the monograph of Paterson [58].

THEOREM 2.1. The following are equivalent:

(i) $G$ is an amenable group;

(ii) $\mathrm{L}^{1}(G)$ is amenable; and

(iii) $\mathrm{L}^{1}(G)$ is biflat.

The equivalence of (i) and (ii) is a famous and motivating theorem of Johnson [42]. The equivalence of (i) and (iii) is due to Helemskiı [35. Note that since $\mathrm{L}^{1}(G)$ always has a bounded approximate identity, (ii) and (iii) are equivalent. It is well-known that $\mathrm{L}^{1}(G)$, which injects densely into a $\mathrm{C}^{*}$-algebra (say $\mathrm{C}^{*}(G)$ ), is contractible if and only if $G$ is finite.

THEOREM 2.2. $\mathrm{L}^{1}(G)$ is always weakly amenable.

This result is due to Johnson [44; a simpler proof may be found in [15]. Helemskiu [35] proved the biprojectivity result below.

THEOREM 2.3. $\mathrm{L}^{1}(G)$ is biprojective if and only if $G$ is compact.

The characterization of amenability for measure algebras is due to Dales, Ghahramani and Helemskiŭ [11].

THEOREM 2.4. The following are equivalent:

(i) $\mathrm{M}(G)$ is weakly amenable;

(ii) $\mathrm{M}(G)$ admits no point derivations; and

(iii) $G$ is discrete (and hence $\mathrm{M}(G)=\ell^{1}(G)$ ).

It is an immediate consequence that $\mathrm{M}(G)$ is amenable if and only if $G$ is discrete and amenable. For abelian groups, Theorem 2.4 was established by Brown and Moran [5]. We note that since $\mathrm{M}(G)$ is unital, if it is biprojective it is then contractible. Moreover since $\mathrm{M}(G)$ injects densely into a $\mathrm{C}^{*}$-algebra (for example the closure of $\lambda(\mathrm{M}(G))$ in $\mathrm{VN}(G)$ ), $\mathrm{M}(G)$ is contractible if and only if $G$ is finite.

We close this section with a result of Runde 68 .

Theorem 2.5. $\mathrm{M}(G)$ is Connes amenable if and only if $G$ is amenable.

2.2. Fourier and Fourier-Stieltjes algebras. For an abelian locally compact group $G$, we have that $G$ is compact if and only if $\widehat{G}$ is discrete; and, by Pontryagin duality, $G$ is discrete if and only if $\widehat{G}$ is compact. Given this, it was long expected that for general locally compact $G$, the results for $\mathrm{A}(G)$ and $\mathrm{B}(G)$ would parallel those for $\mathrm{L}^{1}(G)$ and $\mathrm{M}(G)$. In particular, Leptin [52] showed that $\mathrm{A}(G)$ has a bounded approximate identity if and only if $G$ is amenable. In particular, if $\mathrm{A}(G)$ is amenable, then we must have 
$G$ amenable. It was, for a long time, thought that the converse must hold. Hence the following result of Johnson [45] was a surprise.

TheOrem 2.6. For the compact group $G=\mathrm{SO}(3), \mathrm{A}(G)$ is not weakly amenable.

This was proved by taking a "twisted" convolution map $u \otimes v \mapsto u * \check{v}: \mathrm{A}(G) \otimes^{\gamma} \mathrm{A}(G) \rightarrow$ $\mathrm{A}(G)$, and identifying its range $\mathrm{A}_{\gamma}(G)$ as a Banach algebra in its own right. A non-zero point derivation was found on $\mathrm{A}_{\gamma}(G)$, which was used to show that $\Delta$ is not essential for $\mathrm{A}(G) \otimes^{\gamma} \mathrm{A}(G) \rightarrow \mathrm{A}(G)$ (see Theorem 1.1. Plymen [60, showed that such a point derivation can be found for $\mathrm{A}_{\gamma}(G)$ of any compact semi-simple Lie group.

The actual characterization of amenability is due to Forrest and Runde [24] (see also a more qualitative version in [71]). We say $G$ is virtually abelian if it admits an abelian subgroup of finite index.

THEOREM 2.7. The following are equivalent:

(i) $\mathrm{A}(G)$ is amenable;

(ii) $\check{\Delta}=\left\{\left(s, s^{-1}\right): s \in G\right\} \in \Omega(G)$, where $\Omega(G)$ denotes the smallest ring of subsets containing all cosets; and

(iii) $G$ is virtually abelian.

Moreover, $\mathrm{B}(G)$ is amenable if and only if $G$ is compact and virtually abelian.

It is interesting to note that operator space techniques were used in the proof (and that this proof is intimately related to Theorem 4.7, below). It seems that (ii) $\Leftrightarrow$ (iii) can be proved by purely group theoretic techniques, though the author knows of no reference for this. The implication (iii) $\Rightarrow(\mathrm{i})$ can be found in [50. We note that if $\mathrm{B}_{r}(G)$ is amenable, it must have a bounded approximate identity whose cluster point must be the constant function 1 ; this means that $\mathrm{B}_{r}(G)=\mathrm{B}(G)$. Contractibility of $\mathrm{A}(G)$ is equivalent to finiteness of $G$; indeed $\mathrm{A}(G)$ injects densely into the commutative $\mathrm{C}^{*}$-algebra $\mathcal{C}_{0}(G)$.

The characterization for weak amenability is not yet entirely known, but we have strong partial results.

THEOREM 2.8. (i) If the connected component of the identity $G_{e}$ of $G$ is abelian, then $\mathrm{A}(G)$ is hyper-Tauberian (hence weakly amenable).

(ii) If $G$ contains a non-abelian connected compact subgroup, then $\mathrm{A}(G)$ is not weakly amenable.

(iii) If $G$ is compact and connected, $\mathrm{A}(G)$ is hyper-Tauberian if and only if $G$ is abelian.

We note that connected [SIN] (small invariant neighbourhood) and [MAP] (maximally almost periodic) groups (see tables in [57]) contain non-abelian connected compact subgroups whenever they, themselves, are non-abelian. Forrest and Runde [24] proved (i) for weak amenability, while Samei [77] proved it for the hyper-Tauberian property. Results (ii) and (iii) were proved in [26]; they follow from the next result in the same article which we give below. See Section 1.3 for notation and terminology.

THEOREM 2.9. Let $G$ be a compact group. Then the following are equivalent:

(i) $G_{e}$ is abelian;

(ii) $\mathrm{A}(G)$ is hyper-Tauberian; (ii') $\Delta$ is spectral for $\mathrm{A}(G) \otimes^{\gamma} \mathrm{A}(G)$;

(iii) $\mathrm{A}(G)$ is weakly amenable; and (iii') $\Delta$ is essential $\mathrm{A}(G) \otimes^{\gamma} \mathrm{A}(G)$. 
The proof of this theorem builds on that of Theorem 2.6 and the associated result of Plymen, and uses the characterization of connected compact groups from [61]. The equivalences (ii) $\Leftrightarrow\left(\right.$ ii'$\left.^{\prime}\right)$ and (iii) $\Leftrightarrow\left(\right.$ iii' $\left.^{\prime}\right)$ are direct applications of Theorem 1.1

QUESTION 2.10. Is it the case that $\mathrm{A}(G)$ is weakly amenable only if $G_{e}$ is abelian? Is the Fourier algebra weakly amenable for any of the groups $\mathrm{SL}_{2}(\mathbb{R})$, the $a x+b$-group, or any of the Heisenberg groups?

Given Theorems 2.4, 2.7 and 2.8, it is reasonable to expect that $\mathrm{B}(G)$ ought to be weakly amenable if and only if $G$ is compact and $G_{e}$ is abelian; compare this with Theorem 3.7 (iii), below.

The best results known for biflatness and biprojectivity are recently due to Runde [73]; though some components of this theorem were developed by Aristov [1].

THEOREM 2.11. (i) If $\mathrm{A}(G)$ is biflat then either (a) $G$ is virtually abelian, or (b) $G$ is non-amenable and does not contain a discrete copy of the free group on two generators.

(ii) If $\mathrm{A}(G)$ is biprojective then $G$ is discrete and one of (a), or (b), in (i) above, holds. Conversely, if (a) holds (and $G$ is discrete) then $\mathrm{A}(G)$ is biflat (biprojective).

It seems unlikely that $\mathrm{A}(G)$ could be biflat for any non-amenable group, but little is known about harmonic analysis on non-amenable groups not containing free groups; see Ol'shanskii and Sapir [56] for an up-to-date treatment of the groups themselves. Much as is the case for $\mathrm{M}(G)$, above, $\mathrm{B}(G)$ is biprojective if and only if $G$ is finite: $\mathrm{B}(G)$ has a commutative $\mathrm{C}^{*}$-algebra, the Eberlein algebra, as its uniform closure in $\mathcal{C}_{b}(G)$.

Results on Connes amenability for $\mathrm{B}(G)$ are also incomplete. The best results published are due to Runde [70].

THEOREM 2.12. (i) If $G$ is either discrete and amenable, or is a product of finite groups, then $\mathrm{B}(G)$ is Connes amenable if and only if $G$ is virtually abelian.

(ii) If $G$ is discrete, then $\mathrm{B}_{r}(G)$ is Connes amenable if and only if $G$ is virtually abelian.

3. Operator amenability properties. It is unfortunate that $\mathrm{A}(G)$ is amenable so rarely. The theory of operator spaces, which developed into its modern form in the 1990s, gives a new perspective to these problems. Banach spaces (or even normed spaces) have clear advantages over simple vector spaces for posing problems in infinite dimensional settings. The main reason is that there are useful theorems regarding properties of bounded linear operators, while little can be said about linear operators alone, in general. The theory of operator spaces and completely bounded maps offers a similar refinement in terms of control, however still leaves us enough morphisms for a usable theory.

3.1. Operator spaces and completely bounded maps. We use, as a standard reference for operator spaces, the book of Effros and Ruan [16]; all results in this section can be found there. We also recommend the book of Paulsen [59].

The axioms of Ruan give a simple abstract definition of operator spaces. Let $\mathcal{V}$ be a $\mathbb{C}$-vector space and for each $n$ in $\mathbb{N}$, let $M_{n}(\mathcal{V})$ denote the space of $n \times n$ matrices with entries in $\mathcal{V}$. An operator space structure is a sequence of norms $\left(\|\cdot\|_{n}: M_{n}(\mathcal{V}) \rightarrow \mathbb{R}^{\geq 0}\right)_{n \in \mathbb{N}}$ 
which satisfy

$$
\text { (OS1): }\left\|\left[\begin{array}{cc}
U & 0 \\
0 & V
\end{array}\right]\right\|_{n+m}=\max \left\{\|U\|_{n},\|V\|_{m}\right\}, \quad(\mathrm{OS} 2):\|\alpha U \beta\|_{n} \leq\|\alpha\|\|U\|_{n}\|\beta\|
$$

where $U \in M_{n}(\mathcal{V}), V \in M_{m}(\mathcal{V})$ and $\alpha, \beta \in M_{n}(\mathbb{C})$, where the latter space is normed as the space of linear operators on an $n$-dimensional Hilbert space. We generally assume that $\left(\mathcal{V},\|\cdot\|_{1}\right)$ is complete, and hence a Banach space. We will call $\mathcal{V}$, equipped with an operator space structure, an operator space. It is easily checked that any closed subspace of $\mathcal{B}(\mathcal{H})\left(\mathcal{H}\right.$ a Hilbert space) is an operator space, where we identify $M_{n}(\mathcal{B}(\mathcal{H})) \cong \mathcal{B}\left(\mathcal{H}^{n}\right)$ in the usual manner.

A linear map between operator spaces $T: \mathcal{V} \rightarrow \mathcal{W}$ is called completely bounded if its amplifications, $\left[v_{i j}\right] \mapsto\left[T v_{i j}\right]: M_{n}(\mathcal{V}) \rightarrow M_{n}(\mathcal{W})$, are uniformly bounded in $n$. These are the natural morphisms of operator spaces. We denote the space of such maps $\mathcal{C B}(\mathcal{V}, \mathcal{W})$. It is itself an operator space via the natural identification $M_{n}(\mathcal{C B}(\mathcal{V}, \mathcal{W})) \cong$ $\mathcal{C B}\left(\mathcal{V}, M_{n}(\mathcal{W})\right),\left[T_{i j}\right] \cong\left(v \mapsto\left[T_{i j} v\right]\right)$. All bounded linear functionals are automatically completely bounded, thus $\mathcal{V}^{*}$ is naturally an operator space. In particular $\mathrm{B}(G) \cong \mathrm{C}^{*}(G)^{*}$ is an operator space and $\mathrm{A}(G)$ inherits its operator space structure as a subspace. We note that $\mathrm{M}(G) \cong \mathcal{C}_{0}(G)^{*}$ is a maximal operator space, as is the subspace $\mathrm{L}^{1}(G)$. For a maximal operator space $\mathcal{M}$, any bounded map $T: \mathcal{M} \rightarrow \mathcal{V}$ is automatically completely bounded, whenever it is bounded. In particular, operator space theory does not restrict the natural morphisms emanating from $\mathrm{M}(G)$ or from $\mathrm{L}^{1}(G)$.

A bilinear map $B: \mathcal{V} \times \mathcal{W} \rightarrow \mathcal{Z}$ on operator spaces is called jointly completely bounded if the amplifications $B^{(n, m)}: M_{n}(\mathcal{V}) \times M_{m}(\mathcal{W}) \rightarrow M_{n m}(\mathcal{Z})$, given by $B^{(n, m)}\left(\left[v_{i j}\right],\left[w_{k l}\right]\right)=$ $\left[B\left(v_{i j}, w_{k l}\right)\right]$, are uniformly bounded in pairs $(n, m)$. The operator projective tensor product $\mathcal{V} \widehat{\otimes} \mathcal{W}$ is the canonical object which linearizes jointly completely bounded bilinear maps into completely bounded bilinear maps. A remarkable result, proved by Effros and Ruan, is a Grothendieck-type identification of the operator space projective tensor product of the preduals of two von Neumann algebras: if $\mathcal{M}$ and $\mathcal{N}$ are von Neumann algebras with preduals $\mathcal{M}_{*}$ and $\mathcal{N}_{*}$, then $\mathcal{M}_{*} \widehat{\otimes} \mathcal{N}_{*} \cong(\mathcal{M} \bar{\otimes} \mathcal{N})_{*}$, the predual of the von Neumann tensor product of $\mathcal{M}$ and $\mathcal{N}$. The author does not know of any method of proving this identity without appeal to Tomita-Takesaki theory, in particular the commutation formula $(\mathcal{M} \bar{\otimes} \mathcal{N})^{\prime} \cong \mathcal{M}^{\prime} \bar{\otimes} \mathcal{N}^{\prime}$. For locally compact groups $G$ and $H$ this gives rise to the identification

$$
\mathrm{A}(G) \widehat{\otimes} \mathrm{A}(H) \cong \mathrm{A}(G \times H) .
$$

This contrasts with the result of Losert [54, which was proved using techniques of subhomogeneous von Neumann algebras, that $\mathrm{A}(G) \otimes{ }^{\gamma} \mathrm{A}(H) \cong \mathrm{A}(G \times H)$ if and only if at least one of $G$ or $H$ is virtually abelian. Recall the role of virtually abelian groups in Section 2.2 . These results suggest that the recognition of the natural operator space structures on $\mathrm{A}(G)$ and on $\mathrm{B}(G)$ should play a role in gaining a more satisfactory amenability theory. For example, using Losert's result, Forrest and Wood [29] showed that all bounded maps on $\mathrm{A}(G)$ are automatically completely bounded exactly when $G$ is virtually abelian. 
To this end, an algebra $\mathcal{A}$, which is also an operator space, is called a completely contractive Banach algebra if the multiplication map $m: \mathcal{A} \times \mathcal{A} \rightarrow \mathcal{A}$ is jointly completely contractive, i.e. all amplifications $m^{(n, k)}$ are contractions. An $\mathcal{A}$-bimodule, $\mathcal{V}$, which is also an operator space, is a completely contractive $\mathcal{A}$-bimodule provided the module actions $\mathcal{A} \times \mathcal{V}, \mathcal{V} \times \mathcal{A} \rightarrow \mathcal{V}$ are both jointly completely contractive, which is the same as having that each of the module-defining homomorphism and anti-homomorphism from $\mathcal{A}$ into $\mathcal{C B}(\mathcal{V})$ is completely contractive. If $\mathcal{V}$ is such a module, then $\mathcal{V}^{*}$ is a dual completely contractive $\mathcal{A}$-bimodule. The entire theory of amenability, contractibility, weak amenability, biflatness and biprojectivity (in the case of a function algebra, hyper-Tauberianness) can be adapted to be rephrased with completely bounded derivations, operator projective tensor products, or module maps (or local maps), and turned into the theories of operator amenability, operator weak amenability, etc. In short, the qualifier "operator" means that we convert all results from the Banach space setting into the operator space one. Note that the maximal operator spaces structures on $\mathrm{L}^{1}(G)$ and $\mathrm{M}(G)$ allow the following important fact.

REMARK 3.1. Every Banach algebra theorem on $\mathrm{L}^{1}(G)$ or on $\mathrm{M}(G)$ is automatically a completely contractive Banach algebra theorem.

In other words, all of the results of Section 2.1 are really operator amenability results.

3.2. Fourier and Fourier-Stieltjes algebras as operator spaces. Now, with operator spaces in hand, we can state the true analogues of the theorems of Section 2.1. We let $G$ denote a locally compact group. The seminal theorem is by Ruan 63.

THEOREM 3.2. $\mathrm{A}(G)$ is operator amenable if and only if $G$ is amenable.

The success of this result has inspired constructions of operator space structures on Herz-Figà-Talamanca algebras $\mathrm{A}_{p}(G)$ to gain similar operator amenability results: two quite different constructions are obtained by Lambert et al. [49] and Daws [12. The only systematic attempt, of which the author is aware, to generalize Theorems 2.1 and 3.2 to a locally compact quantum group or Kac algebra setting is by Aristov 2 .

Theorem 3.2 is the appropriate dual analogue to (i) $\Leftrightarrow($ ii) of Theorem 2.1. Since the dense inclusion $\mathrm{A}(G) \hookrightarrow \mathcal{C}_{0}(G)$ is completely contractive, operator contractibility of $\mathrm{A}(G)$ implies that of $\mathcal{C}_{0}(G)$. Since each maximal ideal is co-dimension 1, the arguments of $\S 4.1$ of [66] can be readily adapted to show that $G$ is finite. The facts stated immediately after Theorem 2.1. along with duality considerations, lead to the following.

Conjecture 3.3. $\mathrm{A}(G)$ is always operator biflat.

We do not yet know the truth of this conjecture, but there is strong evidence in its favour. We say that a closed subgroup $H$ in $G$ admits a bounded approximate indicator in $G$ if there is a net $\left(v_{\alpha}\right) \subset \mathrm{B}(G)$ for which

$$
\left.v_{\alpha}\right|_{H} u \rightarrow u \text { for } u \in \mathrm{A}(H) \quad \text { and } \quad v_{\alpha} w \rightarrow 0 \text { for } w \in \mathrm{I}_{\mathrm{A}(G)}(H) .
$$

(See explanation of notation in Section 1.3.) This concept was introduced in [3. It was proved there that bounded approximate indicators can always be built of positive defi- 
nite functions. We observe that they may not be realized in general as elements whose restriction to $H$ is the constant function 1 ; see [47.

We say a locally compact group $Q$ is a [QSIN] (quasi-small invariant neighbourhood) group if $\mathrm{L}^{1}(Q)$ admits a bounded approximate identity $\left(e_{\alpha}\right)$ for which $\left\|e_{\alpha} * \delta_{s}-\delta_{s} * e_{\alpha}\right\|_{1}$ $\rightarrow 0$ for each Dirac measure $\delta_{s}$ in $\mathrm{M}(Q)$.

THEOREM 3.4. (i) If the diagonal subgroup $\Delta$ in $G \times G$ admits a bounded approximate indicator, then $\mathrm{A}(G)$ is operator biflat.

(ii) If $G$ injects continuously into a [QSIN] group, then $\Delta$ admits a bounded approximate indicator in $G \times G$.

(ii) If $G=\mathrm{SL}_{3}(\mathbb{R})$, then $\Delta$ does not admit a bounded approximate indicator in $G \times G$.

This theorem was proved in [3], but we note that Ruan and $\mathrm{Xu} 64$ proved directly that the assumed condition in (ii), above, implies that $\mathrm{A}(G)$ is operator biflat. We do not know if $\mathrm{A}\left(\mathrm{SL}_{3}(\mathbb{R})\right)$ is operator biflat.

We observe that results (ii) and (i), with Leptin's theorem [52, show directly the fact that for an amenable group $G, \mathrm{~A}(G)$ is operator amenable. It is interesting to note that a device in 78 allows us directly to obtain an operator bounded approximate diagonal for $\mathrm{A}(G)$, for amenable $G$. If $G$ is amenable then $G$ is itself [QSIN] (see [55, 79]), and hence, by (ii), there is a bounded approximate indicator $\left(v_{\alpha}\right)$ for $\Delta$ in $G \times G$. Let $\left(u_{\beta}\right)$ be a bounded approximate identity for $\mathrm{A}(G \times G)$. Then

$$
\left(v_{\alpha} u_{\beta}\right) \subset \mathrm{A}(G \times G) \cong \mathrm{A}(G) \widehat{\otimes} \mathrm{A}(G)
$$

and a net $\left(w_{\mu}\right)$ can be extracted from this set which allows limits to be taken first in $\alpha$, then in $\beta$. This net is the desired bounded approximate diagonal.

If Conjecture 3.3 were true, it would imply (i) of the following, which is the dual analogue of Theorem 2.2 .

THEOREM 3.5. (i) $\mathrm{A}(G)$ is always operator weakly amenable.

(ii) $\mathrm{A}(G)$ is always operator hyper-Tauberian.

Part (i) was proved in [78], and independently by Samei [76]. While the proof of [78] relied on the operator space analogue of Theorem 1.1](iii'), that of [76] was the genesis for the hyper-Tauberian condition of [77]. It was there that (ii) was proved; moreover, this result was extended to Herz-Figà-Talamanca algebras $\mathrm{A}_{p}(G)$, using the operator space structure of 49. As mentioned in Section 1.3. (ii) implies (i). An important fact used in both of these proofs is that the subgroup $\Delta$ is spectral for $\mathrm{A}(G \times G)$; see [36, 80. We note that it follows from Theorem 3.5, and the fact that bounded linear functionals are automatically completely bounded, that $\mathrm{A}(G)$ admits no non-zero point derivations.

We have the following dual analogue of Theorem 2.3 which is due to Aristov [1] and, independently, Wood [84].

TheOREM 3.6. $\mathrm{A}(G)$ is operator biprojective if and only if $G$ is discrete.

Daws [13] has recently provided evidence which suggests that Theorems 2.3 and 3.6 are really results on compact Kac algebras, and may not be extended to general compact quantum groups. 
The success of finding the dual analogue of each amenability result about $\mathrm{L}^{1}(G)$ in Section 2.1 for $\mathrm{A}(G)$ (except possibly operator biflatness) led us to think that Theorem 2.4 should admit a dual analogue for $\mathrm{B}(G)$ in the operator space setting. Thus (ii) and (iii) below were a surprise. The operator amenability constant $C_{\mathcal{A}}^{o p}$ is defined for a completely contractive Banach algebra $\mathcal{A}$ analogously to the amenability constant of Section 1.2

THEOREM 3.7. (i) If $\mathrm{B}(G)$ is operator amenable with operator amenability constant $C_{\mathrm{B}(G)}^{o p}<5$, then $G$ is compact.

(ii) There is a class of groups, the "Fell" groups, $G$, for which each $\mathrm{B}(G)$ is operator amenable with $C_{\mathrm{B}(G)}^{o p}=5$.

(iii) For each Fell group $G, \mathrm{~B}(G)$ is weakly amenable.

Part (i) was proved in [74, while parts (ii) and (iii) are from 75. For a Fell group $G$ - an example of which is the semi-direct product $G_{p}=\mathbb{Z}_{p} \ltimes \mathbb{Q}_{p}$ for a prime $p$ - we have the decomposition $\mathrm{B}(G)=\mathrm{A}_{f}(G) \oplus_{\ell^{1}} \mathrm{~A}(G)$. This is a "semi-direct product" of two operator amenable Banach algebras. The lack of the word "operator" in (iii) is not a typographical error. Since a Fell group $G$ is totally disconnected, and so too is its almost periodic compactification - in the case $G_{p}$, above, it is $\mathbb{Z}_{p}$ - both the algebras $\mathrm{A}(G)$ and $\mathrm{A}_{f}(G) \cong \mathrm{A}\left(G^{a p}\right)$ are weakly amenable by Theorem 2.7 (ii).

In particular we notice that for a Fell group, $\mathrm{B}(G)$ has no non-zero point derivations. There are other known examples of this absence of point derivations. If $G$ is a connected semi-simple Lie group, it was shown by Cowling [9] that there is a finite family of closed normal subgroups $N_{1}, \ldots, N_{n}$, with quotient maps $q_{j}: G \rightarrow G / N_{j}$, for which $\mathrm{B}(G)=\ell^{1}-\bigoplus_{j=1}^{n} \mathrm{~B}_{0}\left(G / N_{j}\right) \circ q_{j}$. Moreover, Cowling applied his generalized Kunze-Stein phenomenon [8] to show that each $\mathrm{B}_{0}\left(G / N_{j}\right) / \mathrm{A}\left(G / N_{j}\right)$ is a radical Banach algebra. Since each $\mathrm{A}\left(G / N_{j}\right)$ admits no non-zero point derivations (Theorem 3.5), neither can $\mathrm{B}_{0}\left(G / N_{j}\right)$. These examples stand in marked contrast to (i) $\Leftrightarrow$ (ii) of Theorem 2.4. (The author is grateful to M. Ghandehari for pointing out this example.)

It is further interesting to consider amenability properties of the Rajchman algebras $\mathrm{B}_{0}(G)$. In contrast to the examples above, if $G$ is abelian and non compact, Ghandehari [30] has shown that point derivations exist on $\mathrm{B}_{0}(G)$.

QUeSTION 3.8. (i) When is $\mathrm{B}(G)$ operator amenable? Operator weakly amenable?

(ii) What possible values are there for $C_{\mathrm{B}(G)}^{o p}$ ?

(iii) When is $\mathrm{B}_{0}(G)$ operator amenable? Operator weakly amenable?

(iv) When do $\mathrm{B}(G)$, or $\mathrm{B}_{0}(G)$, admit non-zero point derivations?

In partial answer to (ii), it is suggested in 31 that $C_{\mathrm{B}(G)}^{o p}$ takes only values $4 n+1$. However no example is known to us of examples taking values other than 1 or 5 . It is reasonable to expect that $\mathrm{B}_{0}(G)$ is operator weakly amenable only if $\mathrm{B}_{0}(G)=\mathrm{A}(G)$.

Even when the dual structure is taken into account, the situation for $\mathrm{B}(G)$ is still not clear.

THEOREM 3.9. (i) $\mathrm{B}_{r}(G)$ is operator Connes amenable if and only if $G$ is amenable.

(ii) If $G=\mathrm{F}_{2}$, the free group on two generators, then $\mathrm{B}(G)$ is operator Connes amenable. 
This theorem is proved in 74. The proof of $(\mathrm{i})$ is easy: $\mathrm{B}_{r}(G)$ can be operator Connes amenable only if it is contains the constant function 1 ; conversely if $G$ is amenable, then $\mathrm{A}(G)$ is operator amenable and weak* dense in $\mathrm{B}_{r}(G)$. The proof of (ii) invokes the fact, due to Choi [6], that for $G=\mathrm{F}_{2}, \mathrm{C}^{*}(G)$ is residually finite dimensional. This implies that $\mathrm{A}_{f}(G)$, which is operator amenable, is weak* dense in $\mathrm{B}(G)$. We have, presently, no natural conjectures as to when $\mathrm{B}(G)$ is operator Connes amenable, in general. It is not clear that $\mathrm{B}(G)$ ought to admit an operator normal virtual diagonal in such a case; this condition is not automatic [72].

\section{Applications}

4.1. Ideals of Fourier algebras with bounded approximate identities. It is a natural question, given a Banach algebra, to ask if it is possible to classify its ideals. For an algebra such $\mathrm{A}(G)$, even for $G=\mathbb{T}$, this is a difficult question, since it is known that there are non-spectral subsets (see definition in Section 1.3). Hence one may wish to classify a nice subclass of ideals, say, those admitting bounded approximate identities. For abelian groups this was achieved by Liu et al. [53], using methods of invariant means; this pointed towards the need for some form of averaging in $\mathrm{A}(G)$. Since $\mathrm{A}(G)$ is amenable only for virtually abelian $G$, this points to the necessity to import operator space techniques so we can access arbitrary amenable $G$.

Let $\Omega(G)$ denote the smallest Boolean ring of subsets containing cosets of subgroups, and $\Omega_{c}(G)$ the set of closed elements in $\Omega(G)$. The following is the main result of [23].

THEOREM 4.1. Let $G$ be amenable. Then every closed ideal of $\mathrm{A}(G)$, which has a bounded approximate identity, is of the form $\mathrm{I}_{\mathrm{A}(G)}(E)$ where $E \in \Omega_{c}(G)$.

The proof is based on the fact that an ideal $\mathcal{I}$, in an (operator) amenable Banach algebra $\mathcal{A}$, has a bounded approximate identity if [and only if] it is weakly (completely) complemented. i.e. there is a (completely) bounded projection $P: \mathcal{A}^{*} \rightarrow \mathcal{I}^{\perp}$, the annihilator of $\mathcal{I}$ in $\mathcal{A}^{*}$ (see [35, 66]). This follows from the fact that this projection can be "averaged" to a (completely) bounded $\mathcal{A}$-bimodule projection. Now if $H$ is a closed subgroup of an amenable group $G$, then $\mathrm{VN}(H)$ is an injective von Neumann algebra, from which it can be deduced that there is a completely bounded projection $P: \mathrm{VN}(G) \rightarrow \mathrm{I}_{\mathrm{A}(G)}(H)^{\perp} \cong \mathrm{VN}(H)$. Since, by Theorem $3.2 \mathrm{~A}(G)$ is operator amenable, $P$ can be averaged to invariance (see [83, 28]), hence giving the fact that $\mathrm{I}_{\mathrm{A}(G)}(H)$ admits a bounded approximate identity. Then, careful arguments, using the structure of elements of $\Omega_{c}(G)$, shows that for any $E$ in $\Omega_{c}(G), \mathrm{I}_{\mathrm{A}(G)}(E)$ has a bounded approximate identity.

To see the converse, consider $\mathcal{I}$ a closed ideal in $\mathrm{A}(G)$ with bounded approximate identity $\left(u_{\alpha}\right)$, and let $E=\{s \in G: u(s)=0$ for $u \in \mathcal{I}\}$. Then $\left(u_{\alpha}\right)$ has a weak* (pointwise) cluster point in $\mathrm{B}\left(G_{d}\right)\left(G_{d}\right.$ is $G$ with discrete topology) which is necessarily the indicator function $1_{G \backslash E}$. By Host [38], $E \in \Omega(G)$, and is closed, so $E \in \Omega_{c}(G)$. We moreover show that elements of $\Omega_{c}(G)$ are spectral sets, hence $\mathcal{I}=\mathrm{I}_{\mathrm{A}(G)}(E)$.

We remark that in the setting of Fourier algebras important results leading to Theorem 4.1 were obtained by Forrest [19, 20]. Operator space techniques were applied to this problem for the first time by Ruan and $\mathrm{Xu}$ [64] and Wood [83]. 
In the case that $G$ is discrete, Wood 85 uses operator biprojectivity (Theorem 3.6) to obtain the following.

THEOREM 4.2. If $G$ is discrete, then a closed ideal $\mathcal{I}$ in $\mathrm{A}(G)$ is completely complemented if and only if there is a completely bounded $\mathrm{A}(G)$-bimodule map $P: \mathrm{A}(G) \rightarrow \mathcal{I}$.

In the case that $G$ is amenable it follows that $\mathcal{I}=\mathrm{I}_{\mathrm{A}(G)}(E)$ for some $E$ in $\Omega(G)$. However, for non-commutative free groups $F$ there are certain free sets $E$, due to Leinert [51], which are not elements of $\Omega(F)$, but for which $\mathrm{I}_{\mathrm{A}(F)}(E)$ is completely complemented.

The desire to understand ideals in $\mathrm{A}(G)$ for non-amenable $G$ has led us to consider the algebra $\mathrm{A}_{c b}(G)$, which is the closure of $\mathrm{A}(G)$ in the algebra of completely bounded multipliers $\mathrm{M}_{c b} \mathrm{~A}(G)$; the latter space was introduced by De Canniere and Haagerup [14] and shown to be a dual space. For "weakly amenable" $G$, as defined in [14] (this should not be confused with weakly amenable algebras), we always have that $\mathrm{A}_{c b}(G)$ admits a bounded approximate identity (see [22]). Thus, $\mathrm{A}_{c b}(G)$ represents faithfully on many canonical modules, such as $\mathrm{A}(G)$ and $\mathrm{VN}(G)$, whereas $\mathrm{A}(G)$ does not. It is shown in [14] that $\mathrm{SL}_{2}(\mathbb{R})$ is weakly amenable and that $\mathrm{F}_{2}$, being a finite index subgroup of a lattice in $\mathrm{SL}_{2}(\mathbb{R})$, also enjoys this property. The following result is from [25] and uses techniques similar to those in Theorem 3.9 (ii).

THEOREM 4.3. (i) $\mathrm{M}_{c b} \mathrm{~A}\left(\mathrm{~F}_{2}\right)$ is operator Connes amenable.

(iii) $\mathrm{A}_{c b}\left(\mathrm{~F}_{2}\right)$ is operator amenable.

(iii) Every weakly complemented closed ideal in $\mathrm{A}_{c b}\left(\mathrm{~F}_{2}\right)$ has a bounded approximate identity.

4.2. Fourier algebras of homogeneous spaces. Let $G$ be a locally compact group and $K$ a compact subgroup. The Fourier algebras of the homogeneous spaces $\mathrm{A}(G / K)$ were defined by Forrest [21]: $\mathrm{A}(G / K)=\{u \in \mathrm{A}(G): k \cdot u=u$ for $k \in K\}$, where $k \cdot u(s)=$ $u(s k)$. It was shown in that article that these spaces have many of the accoutrements of operator amenable algebras: for example, they admit bounded approximate identities when $G$ is amenable. Moreover, the analogue of the Grothendieck-Effros-Ruan tensor product formula was shown in [27]: $\mathrm{A}(G / K) \widehat{\otimes} \mathrm{A}(G / K) \cong \mathrm{A}(G / K \times G / K)$ (where, of course $G / K \times G / K$ is the homogeneous space $(G \times G) /(K \times K))$. Hence the following contrast to Theorem 3.2 , proved in [27], was a surprise.

THEOREM 4.4. If $G$ is a compact semi-simple Lie group, then $\mathrm{A}(G \times G / \Delta)$ is not operator weakly amenable.

As an interesting consequence, we could show that for $G$, as in the theorem, $(G \times G \times$ $G \times G) \Delta \times \Delta$ is not spectral for $\mathrm{A}(G \times G \times G \times G)$. We note that the natural identification $s \mapsto(s, e) \Delta$ gives rise to an isomorphic identification $\mathrm{A}(G \times G / \Delta) \cong \mathrm{A}(G)$ only when $G$ is virtually abelian. Otherwise $\mathrm{A}(G \times G / \Delta)$ identifies with a subalgebra $\mathrm{A}_{\Delta}(G)$ of $\mathrm{A}(G)$, which is very similar to the algebra $\mathrm{A}_{\gamma}(G)$ of Johnson [45. Hence Theorem 4.4 is really an analogue of Theorem 2.6 .

We say that $G$ is $[\mathrm{MAP}]_{K}$ if the almost periodic functions on $G$ separate the points on $K$. In [27] we obtained the following results for locally compact $G$. 
THEOREM 4.5. (i) If $G_{e}$ is abelian, then $\mathrm{A}(G / K)$ is hyper-Tauberian, and if $G$ is $[M A P]_{K}$, then $(K \times K) \Delta$ is a set of local synthesis for $\mathrm{A}(G \times G)$.

(ii) If $G$ is amenable and $[M A P]_{K}$, then $\mathrm{A}(G / K)$ is operator amenable if and only if $(K \times K) \Delta \in \Omega(G \times G)$.

If $G$ contains a compact non-abelian connected subgroup $K$, then there is a compact subgroup $K^{*}$ of $G \times G$ for which $\mathrm{A}\left(G \times G / K^{*}\right)$ is not operator weakly amenable; a partial converse to (i). The only cases which we know of where $(K \times K) \Delta \in \Omega(G \times G)$ are when $K$ has a subgroup of finite index which is normal in $G$.

In the follow-up paper [26] we gained an improvement of Theorem 4.4, but with less illustrative methods.

THEOREM 4.6. Let $G$ be a compact group. Then the following are equivalent:

(i) $\mathrm{A}_{\Delta}(G)$ is operator weakly amenable; (i') $\mathrm{A}_{\Delta}(G)$ is weakly amenable;

(ii) $\mathrm{A}_{\Delta}(G)$ is operator hyper-Tauberian; (ii') $\mathrm{A}_{\Delta}(G)$ is hyper-Tauberian;

(iii) $G_{e}$ is abelian.

Moreover, the following are equivalent:

(a) $\mathrm{A}_{\Delta}(G)$ is operator amenable; (a') $\mathrm{A}_{\Delta}(G)$ is amenable;

(b) $G$ is virtually abelian; (b') $\mathrm{A}_{\Delta}(G)=\mathrm{A}(G)$.

What is interesting about this result is that it suggests that $\mathrm{A}_{\Delta}(G)$ contains, in its Banach space structure, critical information about the operator space structure of $\mathrm{A}(G)$.

4.3. Homomorphisms on Fourier algebras. Let $G$ and $H$ be locally compact groups. Cosets of $H$ can be characterized at those subsets $C$ which are closed under the ternary operation $(r, s, t) \mapsto r s^{-1} t$. A map $\alpha: H \rightarrow G$ is called affine if $\alpha\left(r s^{-1} t\right)=\alpha(r) \alpha(s)^{-1} \alpha(t)$ for $r, s, t$ in $C$. We say $\alpha: Y \rightarrow G$ is piecewise affine if there is a partition $Y=\bigcup_{j=i}^{n} Y_{n}$ of $Y$, where each $Y_{j} \in \Omega(H)$ and for each $j$ a coset $C_{j} \supset Y_{j}$ and an affine $\alpha_{j}: Y_{j} \rightarrow G$ for which $\left.\alpha\right|_{Y_{j}}=\left.\alpha_{j}\right|_{Y_{j}}$. We will call $\alpha: Y \subset H \rightarrow G$ a continuous piecewise affine map if each $Y_{j} \in \Omega_{o}(G)$ (the smallest ring of subsets generated by open cosets) and $\alpha$ is continuous. If $u: G \rightarrow \mathbb{C}$ is a function, we can define for piecewise affine $\alpha$, as above, the map $\Phi_{\alpha} u: H \rightarrow \mathbb{C}$ by $\Phi_{\alpha} u(s)=u(\alpha(s))$ if $s \in Y$, and $\Phi_{\alpha} u(s)=0$ if $s \in H \backslash Y$.

The following result was proved, for abelian groups by Cohen [7], generalized to the case that $G$ is virtually abelian by Host 38, and then to the case that $G$ is discrete and amenable by Ilie [40. The definitive form, as presented here, is in [41].

THEOREM 4.7. (i) If $\alpha: Y \subset H \rightarrow G$ is continuous piecewise affine, then $\Phi_{\alpha}: \mathrm{A}(G) \rightarrow$ $\mathrm{B}(H)$ is a completely bounded homomorphism.

(ii) If $G$ is amenable, then every completely bounded homomorphism $\Phi: \mathrm{A}(G) \rightarrow \mathrm{B}(G)$ is of the form $\Phi=\Phi_{\alpha}$, as in (i) above.

(iii) The homomorphism $\Phi_{\alpha}$ maps $\mathrm{A}(G)$ into $\mathrm{A}(H)$ if and only if $\alpha$ is proper, i.e. $\alpha^{-1}(K)$ is compact for each compact $K \subset H$.

As an obvious corollary, we have that if $H$ is connected, only affine $\alpha$ are allowed as "symbols" for completely bounded homomorphisms from $\mathrm{A}(G)(G$ amenable) to $\mathrm{B}(G)$. This theorem seems nearly sharp in the following respects. First, the map $u \mapsto \check{u}: \mathrm{A}(G) \rightarrow$ 
$\mathrm{A}(G)\left(\check{u}(s)=s\left(s^{-1}\right)\right)$ is completely bounded only if $G$ is virtually abelian; compare to Theorem 2.7 (ii) and comments in the paragraph below. Second, if $E$ is a free set in a non-commutative, hence non-amenable, free group $F$, then $u \mapsto 1_{F} u: \mathrm{A}(F) \rightarrow \mathrm{A}(F)$ is completely bounded (see Leinert [51]) but not implemented by a completely bounded symbol (in particular $E \notin \Omega(F)$ ).

Let us illustrate the role of Theorem 3.2 in the proof of (ii). If $\Phi$ is completely bounded, then

$$
\Phi \otimes \operatorname{id}_{\mathrm{A}(G)}: \mathrm{A}(G) \widehat{\otimes} \mathrm{A}(G) \cong \mathrm{A}(G \times G) \rightarrow \mathrm{B}(H \times G)
$$

is well-defined. If $\left(w_{\mu}\right)$ is an operator bounded approximate diagonal for $\mathrm{A}(G)$ of the type whose construction is outlined after Theorem 3.4 then the net $\left(\Phi\left(w_{\mu}\right)\right)$, considered as a bounded net in $\mathrm{B}\left(H_{d} \otimes G_{d}\right)$ (discretized groups) must have a cluster point which is the indicator function $1_{Y}$. Hence $Y \in \Omega(H \times G)$, by Host [38. It can then be checked that $Y$ is the graph of a piecewise affine function $\alpha$, which, moreover, must be continuous.

We define $\alpha: Y \subset H \rightarrow G$ to be mixed piecewise affine if $Y$ partitions as above into elements $Y_{1}, \ldots, Y_{n}$ of $\Omega(H)$, and for $j=1, \ldots, n$ there is a coset $C_{j} \subset Y_{j}$ and either an affine $\alpha_{j}: C_{j} \rightarrow G$, or an anti-affine $\alpha_{j}$ (i.e. $\alpha_{j}\left(r s^{-1} t\right)=\alpha_{j}(t)^{-1} \alpha_{j}(s) \alpha_{j}(s)^{-1}$ ), such that $\left.\alpha\right|_{Y_{j}}=\left.\alpha_{j}\right|_{Y_{j}}$. If $\Phi_{\alpha}$ is defined as above, it is clear that $\Phi_{\alpha}: \mathrm{A}(G) \rightarrow \mathrm{B}(G)$ is a bounded homomorphism.

Conjecture 4.8. If $G$ is amenable then every bounded homomorphism $\Phi: \mathrm{A}(G) \rightarrow$ $\mathrm{B}(H)$ is of the form $\Phi=\Phi_{\alpha}$ for some continuous mixed piecewise affine $\alpha: Y \subset G \rightarrow H$.

It is an interesting open question to characterize completely bounded, never mind bounded, homomorphisms on Fourier algebras of non-amenable groups. As suggested by the proof Theorem 4.7, illustrated above, the completely bounded question is closely linked to our understanding of the idempotents in $\mathrm{M}_{c b} \mathrm{~A}(H \times G)$ (see section 4.1 above for notation).

For group algebras, only the contractive homomorphisms $\Psi: \mathrm{L}^{1}(G) \rightarrow \mathrm{M}(H)$ are well understood; see Greenleaf [33. It would be interesting to see if, for amenable $G$, bounded approximate diagonal methods shed light on the understanding of such bounded homomorphisms. If these structures can be understood, then there is hope for understanding the completely bounded homomorphisms on predual algebras of locally compact quantum groups.

Acknowledgements. The author's research is supported by NSERC, under grant No. 312515-05.

This paper is based on a lecture delivered at the $19^{\text {th }}$ International Conference on Banach Algebras held at Będlewo, July 14-24, 2009. The support for the meeting by the Polish Academy of Sciences, the European Science Foundation under the ESF-EMSERCOM partnership, and the Faculty of Mathematics and Computer Science of the Adam Mickiewicz University at Poznań is gratefully acknowledged.

The author is grateful to E. Samei for reading the manuscript and correcting some errors, and to the referee for correcting many typos and making suggestions to improve the exposition. 


\section{References}

[1] O. Yu. Aristov, Biprojective algebras and operator spaces, J. Math. Sci. (New York) 111 (2002), 3339-3386.

[2] O. Yu. Aristov, Amenability and compact type for Hopf-von Neumann algebras from the homological point of view, in: Banach Algebras and Their Applications, Contemp. Math. 363, Amer. Math. Soc., Providence, RI, 2004, 15-37.

[3] O. Yu. Aristov, V. Runde and N. Spronk, Operator biflatness of the Fourier algebra and approximate indicators for subgroups, J. Funct. Anal. 209 (2004), 367-387.

[4] W. G. Bade, P. C. Curtis, and H. G. Dales, Amenability and weak amenability for Beurling and Lipschitz algebras, Proc. London Math. Soc. 55 (1987), 359-377.

[5] G. Brown and W. Moran, Point derivations on $M(G)$, Bull. London Math. Soc. 8 (1976), $57-64$.

[6] M.-D. Choi, The full $C^{*}$-algebra of the free group on two generators, Pacific J. Math. 87 (1980), 41-48.

[7] P. J. Cohen, On homomorphisms of group algebras, Amer. J. Math.

[8] M. Cowling, The Kunze-Stein phenomenon, Ann. Math. (2) 107 (1978), 209-234.

[9] M. Cowling, The Fourier-Stieltjes algebra of a semisimple group, Colloq. Math. 41 (1979), 89-94.

[10] P. C. Curtis and R. J. Loy, The structure of amenable Banach algebras, J. London Math. Soc. 40 (1989), 89-104.

[11] H. G. Dales, F. Ghahramani and A. Ya. Helemskiǔ, The amenability of measure algebras, J. London Math. Soc. (2) 66 (2002), 213-226.

[12] M. Daws, p-Operator spaces and Figà-Talamanca-Herz algebras, J. Operator Theory 63 (2010), 47-83.

[13] M. Daws, A note on operator biprojectivity of compact quantum groups, arXiv:0905.1935.

[14] J. De Cannière and U. Haagerup, Multipliers of the Fourier algebras of some simple Lie groups and their discrete subgroups, Amer. J. Math. 107 (1985), 455-500.

[15] M. Despić and F. Ghahramani. Weak amenability of group algebras of locally compact groups, Canad. Math. Bull. 37 (1994), 165-167.

[16] E. G. Effros and Z.-J. Ruan, Operator Spaces, London Math. Soc. Monogr. 23, Oxford Univ. Press, 2000.

[17] P. Eymard, L'algèbre de Fourier d'un groupe localement compact, Bull. Soc. Math. France 92 (1964), 181-236.

[18] J. M. G. Fell, Weak containment and induced representations of groups, Canad. J. Math. 14 (1962), 237-268.

[19] B. E. Forrest, Amenability and bounded approximate identities in ideals of $A(G)$, Illinois J. Math. 34 (1990), 1-25.

[20] B. E. Forrest, Amenability and ideals in $A(G)$, J. Austral. Math. Soc. Ser. A 53 (1992), $143-155$.

[21] B. E. Forrest, Fourier analysis on coset spaces, Rocky Mountain J. Math. 28 (1998), 173-190.

[22] B. E. Forrest, Completely bounded multipliers and ideals in $A(G)$ vanishing on closed subgroups, in: Banach Algebras and Their Applications, Contemp. Math. 363, Amer. Math. Soc., Providence, RI, 2004, 89-94.

[23] B. E. Forrest, E. Kaniuth, A. T. Lau, and N. Spronk, Ideals with bounded approximate identities in Fourier algebras, J. Funct. Anal. 203 (2003), 286-304. 
[24] B. E. Forrest and V. Runde, Amenability and weak amenability of the Fourier algebra, Math. Z. 250 (2005), 731-744.

[25] B. E. Forrest, V. Runde and N. Spronk, Operator amenability of the Fourier algebra in the cb-multiplier norm, Canad. J. Math. 59 (2007), 966-980.

[26] B. E. Forrest, E. Samei, and N. Spronk, Weak amenability of Fourier algebras on compact groups, Indiana Univ. Math. J. 58 (2009), 1379-1393.

[27] B. E. Forrest, E. Samei, and N. Spronk, Convolutions on compact groups and Fourier algebras of coset spaces, Studia Math. 196 (2010), 223-249.

[28] B. E. Forrest and N. Spronk, Best bounds for approximate identities in ideals of the Fourier algebra vanishing on subgroups, Proc. Amer. Math. Soc. 134 (2005), 111-116.

[29] B. E. Forrest and P. J. Wood, Cohomology and the operator space structure of the Fourier algebra and its second dual, Indiana Univ. Math. J. 50 (2001), 1217-1240.

[30] M. Ghandehari, Harmonic analysis of Rajchman algebras, Ph.D. Thesis, University of Waterloo, 2010.

[31] M. Ghandehari, H. Hatami and N. Spronk, Amenability constants for semilattice algebras, Semigroup Forum 79 (2009), 279-297.

[32] C. C. Graham and O. C. McGehee, Essays in Commutative Harmonic Analysis, Grundlehren der mathematischen Wissenschaften 238, Springer, New York, 1979.

[33] F. P. Greenleaf, Norm decreasing homomorphisms of group algebras, Pacific J. Math. 15 (1965), 1187-1219.

[34] N. Groenbaek, A characterization of weakly amenable Banach algebras, Studia Math. 94 (1989), 149-162.

[35] A. Ya. Helemskiŭ, The Homology of Banach and Topological Algebras, Mathematics and its Applications (Soviet Series) 41, Kluwer, Dordrecht, 1989.

[36] C. S. Herz, Harmonic synthesis for subgroups, Ann. Inst. Fourier Grenoble 23 (1973), 91-123.

[37] E. Hewitt and K. A. Ross, Abstract Harmonic Analysis I, Grundlehren der mathematischen Wissenschaften 115, Springer, New York, second edition, 1979.

[38] B. Host, Le théorème des idempotents dans $B(G)$, Bull. Soc. Math. France 114 (1986), 215-223.

[39] A. Hulanicki, Groups whose regular representation weakly contains all unitary representations, Studia Math. 24 (1964), 37-59.

[40] M. Ilie, On Fourier algebra homomorphisms, J. Funct. Anal. 213 (2004), 88-110.

[41] M. Ilie and N. Spronk, Completely bounded homomorphisms of the Fourier algebras, J. Funct. Anal. 225 (2005), 480-499.

[42] B. E. Johnson, Cohomology in Banach algebras, Memoirs Amer. Math. Soc. 127 (1972).

[43] B. E. Johnson, Approximate diagonals and cohomology of certain annihilator Banach algebras, Amer. J. Math. 94 (1972), 685-698.

[44] B. E. Johnson, Weak amenability of group algebras, Bull. London Math. Soc. 23 (1991), 281-284.

[45] B. E. Johnson, Non-amenability of the Fourier algebra of a compact group, J. London Math. Soc. 50 (1994), 361-374.

[46] R. V. Kadison, Isometries of operator algebras, Ann. Math. (2) 54 (1951), 325-338.

[47] E. Kaniuth and A. T. Lau, On a separation property of positive definite functions on locally compact groups, Math. Z. 243 (2003), 161-177.

[48] E. Kaniuth and A. T. Lau, Fourier algebras and amenability, in: Banach Algebras and Their Applications, Contemp. Math. 363, Amer. Math. Soc., Providence, RI, 2004, 181192. 
[49] A. Lambert, M. Neufang and V. Runde, Operator space structure and amenability for Figà-Talamanca-Herz algebras, J. Funct. Anal. 211 (2004), 245-269.

[50] A. T.-M. Lau, R. J. Loy and G. A. Willis, Amenability of Banach and $C^{*}$-algebras on locally compact groups, Studia Math. 119 (1996), 161-178.

[51] M. Leinert, Faltungsoperatoren auf gewissen diskreten Gruppen, Studia Math. 52 (1974), 149-158.

[52] H. Leptin, Sur l'algèbre de Fourier d'un groupe localement compact, C. R. Acad. Sci. Paris Sér. A-B 266 (1968), A1180-A1182.

[53] T. S. Liu, A. van Rooij, and J. Wang, Projections and approximate identities for ideals in group algebras, Trans. Amer. Math. Soc. 175 (1973), 469-482.

[54] V. Losert, On tensor products of the Fourier algebras, Arch. Math. (Basel) 43 (1984), $370-372$.

[55] V. Losert and H. Rindler, Asymptotically central functions and invariant extensions of Dirac measure, in: Probability Measures on Groups, VII (Oberwolfach, 1983), Lecture Notes in Math. 1064, Springer, Berlin, 1984, 368-378.

[56] A. Yu. Ol'shanskii and M. V. Sapir, Non-amenable finitely presented torsion-by-cyclic groups, Publ. Math. Inst. Hautes Études Sci. 96 (2003), 43-169.

[57] T. W. Palmer, Banach Algebras and the General theory of *-Algebras. Volume II. *Algebras, Encyclopedia of Mathematics and its Applications 79, Cambridge University Press, Cambridge, 2001.

[58] A. L. T. Paterson, Amenability, Math. Surveys and Monographs 29, Amer. Math. Soc., Providence, RI, 1988.

[59] V. Paulsen, Completely Bounded Maps and Operator Algebras, Cambridge Studies in Advanced Mathematics 78, Cambridge University Press, Cambridge, 2002.

[60] R. J. Plymen, Fourier algebra of a compact Lie group, arXiv:math. FA/0104018.

[61] J. F. Price, Lie Groups and Compact Groups, London Mathematical Society Lecture Note Serie 25, Cambridge Univ. Press, 1977.

[62] H. Reiter and J. D. Stegeman, Classical Harmonic Analysis and Locally Compact Groups, London Mathematical Society Monographs 22, Oxford University Press, 2000.

[63] Z.-J. Ruan, The operator amenability of $A(G)$, Amer. J. Math. 117 (1995), 1449-1474.

[64] Z.-J. Ruan and G. Xu, Splitting properties of operator bimodules and operator amenability of Kac algebras, in: Operator Theory, Operator Algebras and Related Topics (Timişoara, 1996), Theta Found., Bucharest, 1997, 193-216.

[65] V. Runde, Amenability for dual Banach algebras, Studia Math. 148 (2001), 47-66.

[66] V. Runde, Lectures on Amenability, Lecture Notes in Mathematics 1774, Springer, Berlin, 2002 .

[67] V. Runde, Abstract harmonic analysis, homological algebra, and operator spaces, in: Function Spaces (Edwardsville, IL, 2002), Contemp. Math. 328, Amer. Math. Soc., Providence, RI, 2003, 263-274.

[68] V. Runde, Connes-amenability and normal, virtual diagonals for measure algebras. I, J. London Math. Soc. (2) 67 (2003), 643-656.

[69] V. Runde, Applications of operator spaces to abstract harmonic analysis, Expo. Math. 22 (2004), 317-363.

[70] V. Runde, Dual Banach algebras: Connes-amenability, normal, virtual diagonals, and injectivity of the predual bimodule, Math. Scand. 95 (2004), 124-144.

[71] V. Runde, The amenability constant of the Fourier algebra, Proc. Amer. Math. Soc. 134 (2006), 1473-1481. 
[72] V. Runde, A Connes-amenable, dual Banach algebra need not have a normal, virtual diagonal, Trans. Amer. Math. Soc. 358 (2006), 391-402.

[73] V. Runde, Biflatness and biprojectivity of the Fourier algebra, Arch. Math. (Basel) 92 (2009), 525-530.

[74] V. Runde and N. Spronk, Operator amenability of Fourier-Stieltjes algebras, Math. Proc. Cambridge Philos. Soc. 136 (2004), 675-686.

[75] V. Runde and N. Spronk, Operator amenability of Fourier-Stieltjes algebras. II, Bull. Lond. Math. Soc. 39 (2007), 194-202.

[76] E. Samei, Bounded and completely bounded local derivations from certain commutative semisimple Banach algebras, Proc. Amer. Math. Soc. 133 (2005), 229-238.

[77] E. Samei, Hyper-Tauberian algebras and weak amenability of Figà-Talamanca-Herz algebras, J. Funct. Anal. 231 (2006), 195-220.

[78] N. Spronk, Operator weak amenability of the Fourier algebra, Proc. Amer. Math. Soc. 130 (2002), 3609-3617.

[79] R. Stokke, Quasi-central bounded approximate identities in group algebras of locally compact groups, Illinois J. Math. 48 (2004), 151-170.

[80] M. Takesaki and N. Tatsuuma, Duality and subgroups. II, J. Funct. Anal. 11 (1972), 184-190.

[81] M. E. Walter, $W^{*}$-algebras and nonabelian harmonic analysis, J. Funct. Anal. 11 (1972), $17-38$.

[82] J. G. Wendel, Left centralizers and isomorphisms of group algebras, Pacific J. Math. 2 (1952), 251-261.

[83] P. J. Wood, Complemented ideals in the Fourier algebra of a locally compact group, Proc. Amer. Math. Soc. 128 (2000), 445-451.

[84] P. J. Wood, The operator biprojectivity of the Fourier algebra, Canad. J. Math. 54 (2002), $1100-1120$.

[85] P. J. Wood, Invariant complementation and projectivity in the Fourier algebra, Proc. Amer. Math. Soc. 131 (2003), 1881-1890. 
